\title{
论文
}

\section{一种改进的前飞时倾转旋翼机非定常气弹动力学 模型}

\author{
李治权, 夏品奇 ${ }^{*}$ \\ 南京航空航天大学航空宇航学院, 南京 210016 \\ *E-mail:xiapq@nuaa.edu.cn
}

收稿日期: 2017-09-12; 接受日期: 2018-01-18; 网络版发表日期: 2018-07-11

国家自然科学基金(批准号: 11572150)资助项目

摘要 倾转旋翼机前飞时, 旋翼会处于复杂的非定常气动力环境中, 但现有的倾转旋翼机前飞气弹稳定性分析模 型主要采用准定常片条理论对旋翼的空气动力载荷进行气弹动力学建模, 气弹稳定性分析结果与实验值存在明 显误差. 本文采用ONERA非定常气动力模型进行旋翼气动力建模, 并考虑翼型压缩性和失速的影响, 建立了倾 转旋翼机前飞时的非定常气弹动力学模型. 根据NASA试验模型参数, 本文计算了系统各模态频率和阻尼比随前 飞速度的变化, 计算结果相对于现有理论模型更接近试验数据, 表明本文建立的非定常气弹动力学模型可以比现 有模型更准确地描述倾转旋翼机的前飞气弹稳定性.

关键词倾转旋翼机, 前飞, 气弹稳定性, 非定常气动力

\section{1 引言}

倾转旋翼机是一种兼具直升机和螺旋桨飞机飞行 能力的飞行器, 利用安装在机翼端部可以倾转的旋翼 来实现直升机和螺旋桨飞机两种飞行模式的转换，具 有垂直起降和高速巡航飞行等优点. 当飞行到较大速 度时，旋翼桨毂中心偏离平衡位置旋转并产生强烈振 动, 限制了倾转旋翼机前飞速度的提高 ${ }^{[1]}$.

为研究倾转旋翼机的前飞稳定性, Johnson ${ }^{[2]}$ 最初 建立了九自由度简化分析模型，假设模型为铰接式旋 翼、刚性短舱和弹性机翼的耦合系统，结构模型包括 旋翼和机翼的低阶模态，对倾转旋翼机前飞失稳速度
边界和影响参数进行研究. Nixon ${ }^{[3,4]}$ 采用有限元方法 对悬臂梁弹性机翼进行建模 ${ }^{[3]}$, 并对含气弹剪裁效应 的复合材料桨叶进行建模 ${ }^{[4]}$. 岳海龙和夏品奇 ${ }^{[5]}$ 建立了 用于倾转旋翼机前飞时动力学稳定性分析的简化理论 模型; 建立了针对含弹性弯扭耦合效应机翼的倾转旋 翼机前飞气弹动力学分析模型 ${ }^{[6]}$, 研究机翼不同弯扭 耦合特性对倾转旋翼机前飞气弹稳定性的影响; 在简 化模型基础上考虑了短舱和机翼之间的弹性连接, 研 究了倾转旋翼机前飞不稳定问题的发生机理和失稳时 桨毂中心的运动形态 ${ }^{[1]}$. Singh等人 ${ }^{[7]}$, Zhang和 Smith ${ }^{[8]}$ 通过主动和被动控制等方法，提高倾转旋翼机的最大 前飞速度. 上述研究中的前飞动力学模型均采用片条

引用格式: 李治权, 夏品奇. 一种改进的前飞时倾转旋翼机非定常气弹动力学模型. 中国科学: 技术科学, 2018, 48: 901-907 Li Z Q, Xia P Q. An improved unsteady aeroelastic model for tiltrotor aircraft in forward flight (in Chinese). Sci Sin Tech, 2018, 48: 901-907, doi: $10.1360 / \mathrm{N} 092017-00314$ 
准定常气动力理论, 并对倾转旋翼机的桨叶和机翼结 构模型进行完善，以改进前飞性能. Kim等人 ${ }^{[9]}$ 通过使 用Theodorsen非定常气动力理论建立系统动力学模型, 并在时域和频域中对系统稳定性进行分析，对倾转旋 翼机前飞失稳边界进行计算和预测.

倾转旋翼机在前飞过程中，尤其是在高速飞行阶 段，旋翼会处在复杂的非定常气动力环境中，现有研 究中所采用的气动力模型难以准确地描述旋翼的非定 常气动力问题. 同时由于倾转旋翼机结构模型包含大 量自由度，各自由度之间还存在相互耦合，复杂的非 定常气动力模型会使气弹动力学模型更为复杂. $\mathrm{ON}-$ ERA 非定常气动力模型 ${ }^{[10,11]}$ 是用于计算翼型的半经验 非线性气动载荷模型，相对于简单的基于准定常片条 理论的气动力模型, ONERA非定常气动力模型更能模 拟出桨叶翼型各剖面的非定常气动力环境；相对于其 他非定常气动力模型, ONERA非定常气动力模型所需 迭代的次数更少. 为满足非定常气动力的计算, 同时兼 顾计算效率和准确率, 本文采用ONERA 非定常气动模 型对倾转旋翼机旋翼气动力进行建模，建立了更为准 确的倾转旋翼机前飞时的非定常气弹动力学模型，进 一步计算并分析Bell倾转旋翼机在前飞时的气弹稳定 性，并将计算结果与现有的基于片条准定常气动力理 论的分析结果和试验数据进行了对比.

\section{2 倾转旋翼机结构动力学建模}

\section{1 旋翼桨叶运动方程}

图1 为倾转旋翼机前飞动力学结构模型, 该模型包 括了弹性机翼、刚性短舱和铰接式旋翼. 短舱的一端与 旋翼系统连接，另一端在点 $P$ 处刚性地安装在弹性机翼 上. 机翼的端部运动包括 6 个自由度, 分别为 3 个平动自 由度 $x_{p}, y_{p}, z_{p}$ 和 3 个转动自由度 $\alpha_{x}, \alpha_{y}, \alpha_{z}$, 短舱支点和桨毂 间的距离为短舱长度 $h$. 根据Johnson ${ }^{[2]}$ 九自由度旋翼模 型的描述, 在旋转坐标系下, 旋翼桨叶运动方程为

$$
\begin{aligned}
I_{\beta}^{*}\left(\ddot{\beta}+v_{\beta}^{2} \beta\right)+I_{\beta \alpha}^{*}\left[-\left(\ddot{\alpha}_{y}-2 \dot{\alpha}_{x}\right) \cos \psi_{m}\right. \\
\left.+\left(\ddot{\alpha}_{x}+2 \dot{\alpha}_{y}\right) \sin \psi_{m}\right]+S_{\beta}^{*} \ddot{z}_{p}=\gamma \frac{M_{F}}{C_{l \alpha} c}, \\
I_{\zeta}^{*}\left(\ddot{\zeta}+v_{\zeta}^{2} \zeta\right)+S_{\zeta}^{*}\left[\left(\ddot{x}_{p}+h \ddot{\alpha}_{y}\right) \sin \psi_{m}\right. \\
\left.\quad-\left(\ddot{y}_{p}-h \ddot{\alpha}_{x}\right) \cos \psi_{m}\right]-I_{\zeta \alpha}^{*} \ddot{\alpha}_{z}=\gamma \frac{M_{L}}{C_{l \alpha} c},
\end{aligned}
$$

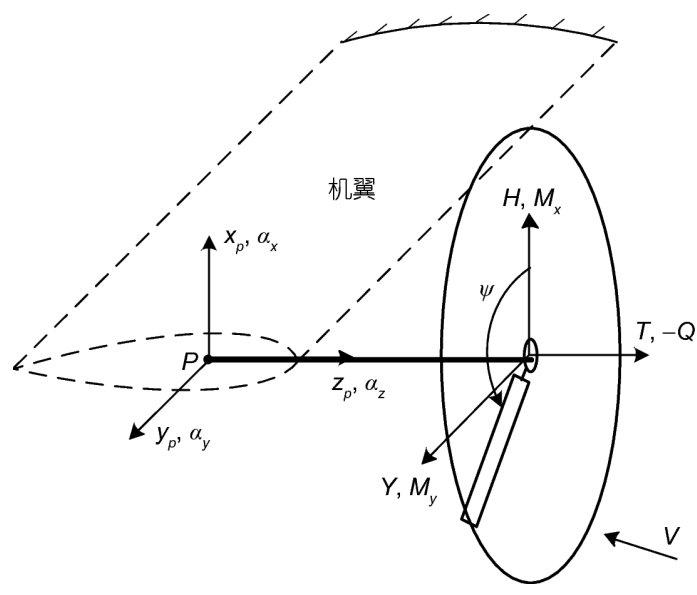

图 1 倾转旋翼机前飞动力学模型

其中, $\gamma, C_{l \alpha}, c$ 分别为桨叶的Lock数、桨叶升力线系 数、桨叶弦长, $v_{\beta}$ 和 $v_{\zeta}$ 分别为桨叶的挥舞和摆振频率, 上述方程用桨叶挥舞惯性矩 $I_{b}$ 进行无因次化 (如 $\left.I_{\beta}^{*}=I_{\beta} / I_{b}\right), M_{F}$ 和 $M_{L}$ 分别表示桨叶挥舞和摆振方向 的非定常气动力矩，并在第 3 节中进行非定常气动力建 模, $\psi_{m}$ 为桨叶方位角, 对方程(1)和(2)进行傅里叶变换, 可将上述方程转换到固定坐标系下的旋翼运动方程 ${ }^{[12]}$.

\section{2 弹性机翼/短舱动力学建模}

在文本推导中, 将机翼/短舱系统视为端部带集中质 量的弹性欧拉梁，刚性短舱通过质量耦合的方式与机翼 连接，并采用有限元离散方法对弹性机翼进行结构动力 学建模. 对于弹性机翼, 其广义Hamilton原理可表示为

$\delta \Pi=\int_{t_{1}}^{t_{2}}\left(\delta U_{w}-\delta T_{w}-\delta W_{w}\right) \mathrm{d} t=0$,

式中, $\delta U_{w}, \delta T_{w}$ 和 $\delta W_{w}$ 分别表示机翼段的虚应变能、虚动 能和外力虚功的变分. 如图2所示，距离机翼根部 $x$ 处的 机翼截面上某点速度 $\overrightarrow{V_{w}}$ 在惯性系下的各速度分量分 别为

$$
\begin{aligned}
& V_{w x}=\dot{u}_{w}, \\
& V_{w y}=\dot{v}_{w}, \\
& V_{w z}=\dot{w}_{w}+y \dot{\phi}_{w},
\end{aligned}
$$

式中, $u_{w}, v_{w}, w_{w}$ 和 $\phi_{w}$ 分别为机翼沿展向、弦向、垂直 方向的线变形和扭转变形, $y$ 为机翼扭转轴和机翼剖面 重心的距离. 根据广义Hamilton原理, 弹性机翼的虚动 


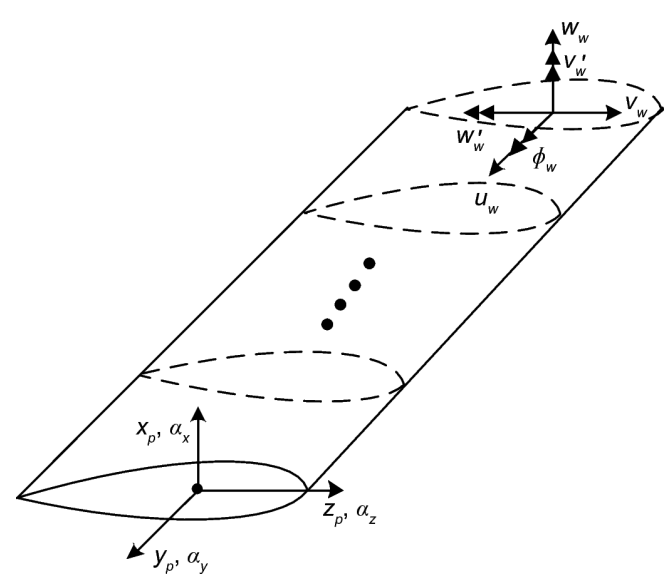

图 2 机翼结构动力学模型

能为

$\delta T_{w}=\int_{0}^{y_{t w}} m_{w} \vec{V}_{w} \cdot \delta \vec{V}_{w} \mathrm{~d} x+m_{p} \vec{V}_{w} \cdot \delta \vec{V}_{w}$,

式中, $m_{w}$ 为机翼单位长度质量, $m_{p}$ 为短舱质量, $y_{t w}$ 为机 翼长度. 机翼段采用弹性悬臂梁方法进行有限元离散 处理, 并采用准定常片条理论进行气动力建模, 虚位 能 $\delta U_{w}$ 和气动力虚功 $\delta W_{w}$ 可参见文献[4], 并将各虚能量 代入到Hamilton表达式(3)中，合并相同变量项并整理 成矩阵形式如下所示:

$\mathbf{M}_{w} \ddot{\mathbf{q}}_{w}+\mathbf{C}_{w} \dot{\mathbf{q}}_{w}+\mathbf{K}_{w} \mathbf{q}_{w}=\mathbf{F}_{w}$,

式中, $\mathbf{q}_{w}$ 为机翼各有限元段自由度所组成的列向 量, 即:

$\mathbf{q}_{w}=\left\{u_{w}^{1}, w_{w}^{1}, v_{w}^{1}, \phi_{w}^{1}, v^{\prime 1}, w_{w}^{\prime 1}, \ldots\right.$,

$$
\left.u_{w}^{n}, w_{w}^{n}, v_{w}^{n}, \phi_{w}^{n}, v_{w}^{\prime \prime}, w_{w}^{\prime \prime}\right\}^{\mathrm{T}} \text {. }
$$

本文将弹性机翼离散成 6 个有限元节点 $(n=6)$, 不 考虑机翼的前掠角, 机翼端部的弹性梁变形自由度与 方程(1)和(2)中机翼端部与桨毂相连的6个自由度之间 存在如下关系:

$$
\left[\begin{array}{c}
x_{p} \\
y_{p} \\
z_{p} \\
\alpha_{x} \\
\alpha_{y} \\
\alpha_{z}
\end{array}\right]=\left[\begin{array}{cccccc}
\cos \alpha_{p} & 0 & -\sin \alpha_{p} & 0 & 0 & 0 \\
0 & 1 & 0 & 0 & 0 & 0 \\
\sin \alpha_{p} & 0 & \cos \alpha_{p} & 0 & 0 & 0 \\
0 & 0 & 0 & \cos \alpha_{p} & 0 & -\sin \alpha_{p} \\
0 & 0 & 0 & 0 & 1 & 0 \\
0 & 0 & 0 & \sin \alpha_{p} & 0 & \cos \alpha_{p}
\end{array}\right]
$$

$$
\left.\times\left[\begin{array}{cccccc}
0 & 1 & 0 & h & 0 & 0 \\
1 & 0 & 0 & 0 & -h & 0 \\
0 & 0 & 1 & 0 & 0 & 0 \\
0 & 0 & 0 & 0 & 1 & 0 \\
0 & 0 & 0 & 1 & 0 & 0 \\
0 & 0 & 0 & 0 & 0 & 1
\end{array}\right] \begin{array}{c}
u_{\text {tip }} \\
w_{\text {tip }} \\
v_{\text {tip }} \\
\phi_{\text {tip }} \\
v_{\text {tip }}^{\prime} \\
w_{\text {tip }}^{\prime}
\end{array}\right] .
$$

通过式(7)可实现旋翼动力学方程与机翼动力学 方程在机翼端部的组装.

\section{3 旋翼非定常气动力建模}

倾转旋翼机在高速飞行过程中, 翼型的气动力变 化是一个复杂的动态过程. 本文采用ONEAR非定常气 动力模型 ${ }^{[10,11]}$ 对倾转旋翼机旋翼进行气动力建模, 桨 叶剖面翼型上的气动力方向如图3所示.

图3中, $V$ 表示翼型上空气来流速度, $\alpha$ 为翼型迎角 迎角, $L_{y}$ 和 $L_{z}$ 分别为翼型非定常弦向气动力和法向气 动力, 根据ONERA气动力模型的描述 ${ }^{[10,11]}$, 翼型上的 非定常气动力包括环量载荷、非环量载荷和型阻 3 个 部分, 其中环量载荷表示为

$$
\begin{aligned}
& L_{y c}=-q c \frac{w_{b 0}-\lambda_{w 0}}{v_{2 d e}}\left[c_{l \alpha} \sin \alpha-\frac{c_{l \alpha} w_{b 1}}{2 v_{2 d e}}\right], \\
& L_{z c}=q c \frac{V}{v_{2 d e}}\left(c_{l a} \sin \alpha\right), \\
& M_{x c}=\frac{1}{4} c L_{z c},
\end{aligned}
$$

式中, $w_{b i}(i=1,2,3)$ 代表由桨叶运动引起的法向速度, 包括桨叶由于挥舞引起的垂向速度和前飞速度, $\lambda_{w 0}$ 为 旋翼引起的诱导入流, 在倾转旋翼机前飞过程中, 旋翼 处于轴流状态, 因此诱导入流 $\lambda_{w 0}$ 可忽略. $c$ 为翼型弦长, $q$ 为动压, 定义为 $q=\frac{1}{2} \rho v_{3 d}^{2}$, 其中 $\rho$ 为空气密度, $v_{3 d}$ 为翼 型合速度, 即 $v_{3 d}=\sqrt{u^{2}+v^{2}+w^{2}}$. 翼型非环量气动力来

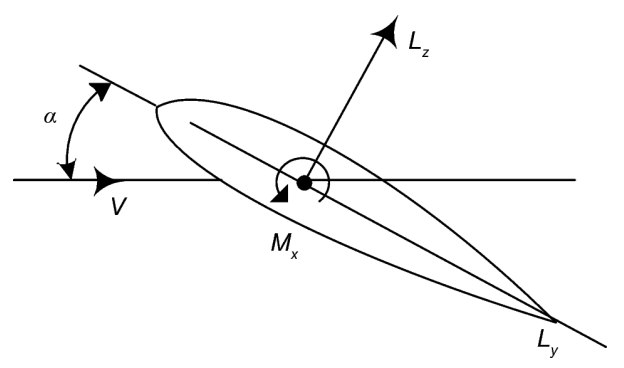

图 3 桨叶剖面气动力分量示意图 
自翼型在平衡位置振动时所产生的气动载荷，可表 示为

$L_{z n c}=\frac{1}{4} \rho c^{2} \pi\left(\dot{w}_{b 0}\right)$,

$M_{x n c}=-\frac{1}{64} \rho c^{3} \pi\left(\dot{w}_{b 1}\right)$.

翼型型阻载荷项可以表示为

$L_{x d}=\frac{1}{2} \rho c c_{d} u v_{3 d}$,

$L_{y d}=\frac{1}{2} \rho c c_{d} v v_{3 d}$,

$L_{z d}=\frac{1}{2} \rho c c_{d} w v_{3 d}$,

式中, $C_{d}$ 为翼型阻力系数.

由于倾转旋翼机可进行高速飞行，旋翼部分翼型 会出现失速和超声速现象, 需要对所建立的气动力模 型进行修正: 式(8)中的升力线斜率 $C_{l a}$ 受当地马赫数的 影响, 即 $C_{l a}=C_{l a s}\left(1-M a^{2}\right)^{-0.5}$, 其中 $C_{l a s}$ 为静态的二维升 力线斜率, $M a$ 为翼型当地马赫数, 当翼型当地马赫数 接近声速时, 阻力和扭转力矩急剧增大, 升力线斜率迅 速减小. 根据文献[2], 当桨叶迎角小于 12 时, 阻力系数 为 $C_{d}=0.0065-0.021 \alpha+0.4 \alpha^{2}$; 当桨叶迎角大于 $12^{\circ}$ 时, 阻 力系数为 $C_{d}=2(\sin \alpha)^{2}$, 将修正后的升力线斜率和阻力 系数带入到气动力表达式(8) (10)中, 对气动力模型进 行修正.

将上述各方向经修正后的桨叶微段气动力分别沿 桨叶径向积分, 进行无因次化得到气动力矩, 其中方程 (1)和(2)中的非定常气动力矩分别为

$\frac{M_{F}}{C_{l a} c}=\int_{0}^{1} r \frac{L_{z}}{C_{l a} c} \mathrm{~d} r$,

$\frac{M_{L}}{C_{l a c} c}=\int_{0}^{1} r \frac{L_{y}}{C_{l a} c} \mathrm{~d} r$,

式中, $L_{z}=L_{z c}+L_{z n c}+L_{z d}$ 以及 $L_{y}=L_{y c}+L_{y n c}$.

\section{4 前飞时气弹稳定性求解方法}

将上文推导的非定常气动力表达式(11)代入到动 力学方程(1)和(2)右端, 经傅里叶变换后, 可得到倾转 旋翼机前飞时的运动微分方程:

$\mathbf{M} \ddot{\mathbf{x}}+\mathbf{C}(\psi) \dot{\mathbf{x}}+\mathbf{K}(\psi) \mathbf{x}=\mathbf{F}$,

式中, $\mathbf{M}, \mathbf{C}, \mathbf{K}$ 分别是系统的质量、阻尼和刚度矩阵, $\mathbf{x}$
为系统各模态组成的列向量，右端 $\mathbf{F}$ 包括外力项、非 线性项和与变量无关的常数项. 本文采用 3 片铰接式 桨叶旋翼, 弹性机翼离散成 6 个有限元节点, 则 $\mathbf{x}$ 定义为

$$
\begin{aligned}
\mathbf{x}=\left\{x_{R}, x_{w}\right\}^{\mathrm{T}}=\left\{\beta_{0}, \beta_{1 c}, \beta_{1 s}, \xi_{0}, \xi_{1 c}, \xi_{1 s}, u_{w}^{1}, v_{w}^{1},\right. \\
\left.\phi_{w}^{1}, v^{\prime}{ }_{w}^{1}, w^{\prime \prime}{ }_{w}^{1}, \ldots, u_{w}^{6}, v_{w}^{6}, \phi_{w}^{6}, v^{\prime}{ }_{w}^{6}, w^{\prime 6}{ }_{w}^{6}\right\}^{\mathrm{T}} .
\end{aligned}
$$

由于方程(12)左端存在非线性项，需要对其进行 线性化处理. 旋翼的非定常气动力来自于翼型在平衡 位置的振动, 所以要完成方程的线化, 需要首先确定 各模态在指定飞行状态下的平衡值. 本文采用风洞配 平的方法计算倾转旋翼机在不同前飞速度时的模型配 平值，首先通过计算在指定桨盘升力系数下各变距量 随时间响应确定配平操纵量 ${ }^{[13]}$, 将方程(12)中关于配 平操纵量 $\theta_{0}, \theta_{c}$ 和 $\theta_{s}$ 的项移至方程左端，并引入配平控 制方程如下:

$$
\begin{aligned}
& {\left[\begin{array}{ccc}
\tau_{0} & 0 & 0 \\
0 & \tau_{1} & 0 \\
0 & 0 & \tau_{1}
\end{array}\right]\left[\begin{array}{c}
\theta_{0} \\
\theta_{s} \\
\theta_{c}
\end{array}\right)^{*}+\left(\begin{array}{c}
\theta_{0} \\
\theta_{s} \\
\theta_{c}
\end{array}\right)=\left[\begin{array}{ccc}
K_{0} & 0 & 0 \\
0 & K_{1} & 0 \\
0 & 0 & K_{1}
\end{array}\right] } \\
& \times\left[\begin{array}{ccc}
\frac{6}{C_{l \alpha}} & 0 & 0 \\
0 & \frac{8\left(v_{\beta}^{2}-1\right)}{\gamma} & -1 \\
0 & 1 & \frac{8\left(v_{\beta}^{2}-1\right)}{\gamma}
\end{array}\right]\left[\begin{array}{c}
\bar{C}_{T}-C_{T} \\
\bar{\beta}_{s}-\beta_{s} \\
\bar{\beta}_{c}-\beta_{c}
\end{array}\right],
\end{aligned}
$$

式中, $\tau_{0}, \tau_{1}$ 和 $K_{0}, K_{1}$ 均为控制配平计算收玫速度的参数, $\bar{C}_{T}, \bar{\beta}_{s}$ 和 $\bar{\beta}$ 分别为目标配平函数. 结合方程(12)和(14) 构建配平动力学方程如下:

$\mathbf{M}_{c} \ddot{\mathbf{x}}_{c}+\mathbf{C}_{c} \dot{\mathbf{x}}_{c}+\mathbf{K}_{c} \mathbf{x}_{c}=\mathbf{F}_{c}$,

式中, $\mathbf{x}_{c}=\left\{x_{R}, x_{w}, x_{\theta}\right\}^{\mathrm{T}}, x_{\theta}$ 包含旋翼的总距和周期变距, 通过计算方程(15)在零初始条件下各操纵量随时间响 应确定旋翼配平所需要操纵量，并代入到方程(12)中 计算各模态随时间响应，从而得到不同前飞速度时各 模态的平衡值.

通过计算得到不同前飞速度时各模态的平衡值 后, 本文采用小扰动假设方法进行方程线性化处理, 每 个模态可写成在平衡位置的稳态值和扰动值之和, 即

$$
\begin{aligned}
& x_{R}=x_{R 0}+\Delta x_{R}, \\
& x_{w}=x_{w 0}+\Delta x_{w} .
\end{aligned}
$$

将式(16)代入到方程(12)左端，展开后消去高阶扰 
动项并移至方程左边进行整理，可得到用于倾转旋翼 机前飞稳定性分析的扰动方程:

$\mathbf{M} \Delta \ddot{x}+\mathbf{C}_{0} \Delta \dot{x}+\mathbf{K}_{0} \Delta x=0$,

式中, $\mathbf{C}_{\mathbf{0}}$ 和 $\mathbf{K}_{\mathbf{0}}$ 分别为线化之后的阻尼和刚度矩阵.

方程(17)中阻尼和刚度矩阵中含有随桨叶方位角 周期变化的系数, 并满足如下关系:

$C_{0}\left(\psi_{0}\right)=C_{0}\left(\psi_{0}+2 \pi\right)$,

$K_{0}\left(\psi_{0}\right)=K_{0}\left(\psi_{0}+2 \pi\right)$.

本文应用Floquet理论对含周期系数的动力学方程 进行分析, 将方程(17)转化成状态空间下的一阶微分 方程, 即

$\dot{\mathbf{Y}}=\mathbf{A}(\psi) \mathbf{Y}$,

式中, $\mathbf{Y}=\left\{\Delta x_{R}, \Delta \dot{x}_{R}, \Delta x_{w}, \Delta \dot{x}_{w}\right\}, \mathbf{A}(\psi)$ 为周期函数组成 的状态矩阵，根据Floquet理论，本文采用四阶龙格-库 塔法计算方程(19)在一个周期内的全部响应，得到状 态转移矩阵 $\left[\boldsymbol{\Phi}\left(t, t_{0}\right)\right]$, 并求出 $\left[\boldsymbol{\Phi}\left(t, t_{0}\right)\right]$ 在一个周期端点 $\left(t=t_{0}+2 \pi\right)$ 值的全部特征值 $\lambda$ 和特征向量, 系统模态频率 $\omega$ 和模态阻尼比 $\zeta$ 由下式求出:

$\omega=\operatorname{Im}(\lambda)$,

$\zeta=-\operatorname{Re}(\lambda) /|\lambda|$.

若系统某一模态阻尼比小于 0 , 即表示该模态进入 不稳定状态, 系统发散. 当系统首次出现某一模态阻尼 比等于 0 时所对应的前飞速度定义为倾转旋翼机前飞 临界失稳速度.

\section{5 算例分析与模型验证}

本文算例数据采用NASA的Bell倾转旋翼机试验 模型参数 ${ }^{[2]}$, 旋翼和机翼的主要参数见表 1.

本文计算系统各模态频率和各机翼模态阻尼比随 前飞速度变化曲线, 如图4和5所示. 图4中, $p, q_{1}$ 和 $q_{2}$ 分 别为机翼扭转、垂向弯曲和弦向弯曲基阶模态. 本文 将系统各模态频率和机翼各模态阻尼比的计算结果与 现有的基于准定常气动力的Johnson模型和Nixon模型 的计算结果进行对比, 并与NASA倾转旋翼机半展结 构全尺寸风洞试验结果进行对比. 根据文献[2]的描述, 该试验是通过给予机翼端部初始激励，根据机翼端部 的瞬态衰减运动确定频率和阻尼比, 主要用于测量机
表 1 倾转旋翼机试验模型主要参数

\begin{tabular}{|c|c|c|c|}
\hline \multicolumn{3}{|c|}{ 参数 } & 数值 \\
\hline \multirow{8}{*}{ 旋翼参数 } & \multicolumn{2}{|c|}{ 旋翼半径 (m) } & 3.82 \\
\hline & \multicolumn{2}{|c|}{ 桨叶片数 } & 3 \\
\hline & \multicolumn{2}{|c|}{ 旋翼转速 $(\mathrm{r} / \mathrm{min})$} & 458 \\
\hline & \multicolumn{2}{|c|}{ 翼型 } & NACA23012 \\
\hline & \multicolumn{2}{|c|}{ 桨叶洛克数 } & 3.83 \\
\hline & \multicolumn{2}{|c|}{ 旋翼实度 } & 0.089 \\
\hline & \multicolumn{2}{|c|}{ 静态升力线系数 } & 5.7 \\
\hline & \multicolumn{2}{|c|}{ 桨叶挥舞惯性矩 $\left(\mathrm{kg} / \mathrm{m}^{2}\right)$} & 142 \\
\hline \multirow{9}{*}{ 机翼参数 } & \multicolumn{2}{|c|}{ 机翼展长 $(\mathrm{m})$} & 5.09 \\
\hline & \multirow{3}{*}{$\begin{array}{c}\text { 机翼结构刚度 } \\
\left(\mathrm{kg} \mathrm{m}^{2} \mathrm{~s}^{-2}\right)\end{array}$} & 垂向 & $9.2 \times 10^{6}$ \\
\hline & & 弦向 & $2.5 \times 10^{7}$ \\
\hline & & 扭转 & $1.8 \times 10^{6}$ \\
\hline & \multirow{3}{*}{$\begin{array}{c}\text { 机翼结构阻尼 } \\
\left(\mathrm{kg} \mathrm{m}^{2} \mathrm{~s}^{-2}\right)\end{array}$} & 垂向 & 9030 \\
\hline & & 弦向 & 27300 \\
\hline & & 扭转 & 955 \\
\hline & \multicolumn{2}{|c|}{ 短舱长度 (m) } & 1.31 \\
\hline & \multicolumn{2}{|c|}{ 短舱绕弾性轴惯量 $\left(\mathrm{kg} / \mathrm{m}^{2}\right)$} & 16380 \\
\hline
\end{tabular}

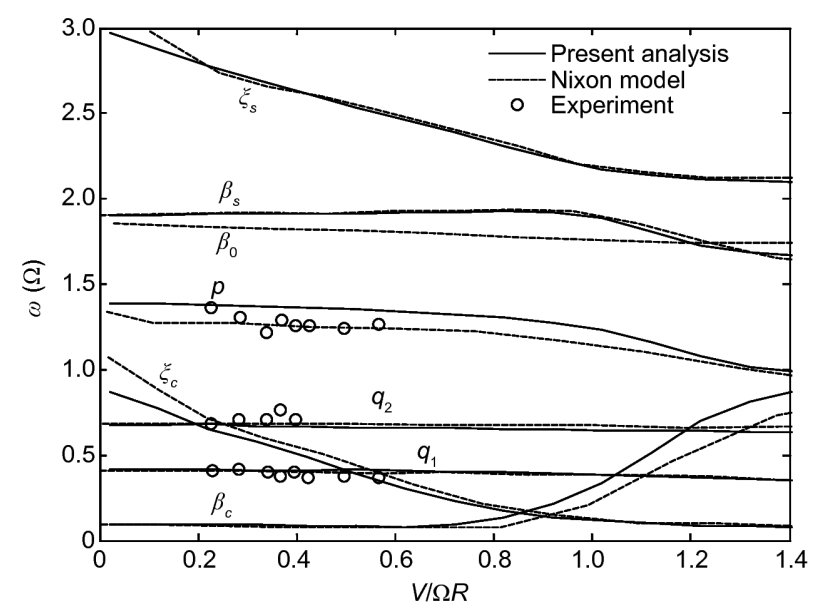

图 4 各模态频率随前飞速度变化曲线

翼垂向弯曲模态的动力学特性, 试验风洞最大吹风速 度约 $400 \mathrm{~km} / \mathrm{h}, V / \Omega R=0.6$.

从图4可以看出, 各理论模型的模态频率计算值之 间的差异较小, 机翼各模态频率计算值与试验值较吻 合. 从图 5 可以看出, 各理论模型的机翼阻尼比计算值 之间存在明显差异, 主要是由于不同的结构模型和气 动力模型影响桨盘平面各气动力, 进而对各模态阻尼 比产生影响. 从图5(a)可以看出, 基于片条准定常气动 

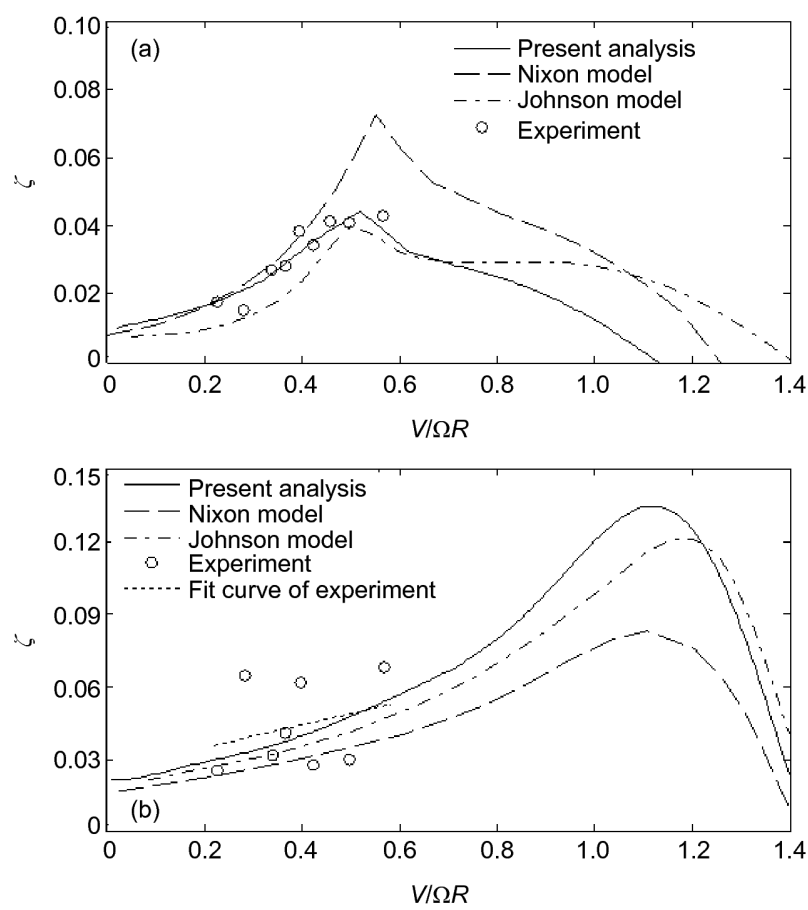

图 5 机翼垂向模态(a)和扭转模态(b)阻尼比随前飞速度变 化曲线

力模型的Nixon模型和Johnson模型的计算值与试验值 相差都较大, 本文基于ONERA 非定常气动力模型的机 翼垂向模态阻尼比的计算值与试验值很接近. 从 图5(b)可以看出, 机翼扭转模态各阻尼比的试验值较 为分散, 为方便比较, 本文对离散的机翼扭转模态阻尼 比的试验值进行多项式曲线拟合，如图5(b)所示，可以 看出，本文基于ONERA非定常气动力模型的机翼扭转 模态阻尼比的计算值与试验值的拟合曲线最接近，各 理论计算值与试验值所存在的误差一方面来自于不同
的气动力模型, 另一方面由于各理论模型均采用悬臂欧 拉梁的理论模型对机翼部分进行建模, 不能完整地反映 试验所使用的真实机翼的结构特性, 造成计算误差. 这 些计算值与试验值的比较, 说明本文所建立的气弹动力 学模型能准确地分析倾转旋翼机的前飞稳定性, 在前飞 时旋翼所处的非定常气动力环境不可忽视. 从图 $5(\mathrm{a})$ 还 可以看出，本文所计算的倾转旋翼机前飞临界失稳速 度为 $1.12 \Omega R$, 小于其余理论模型的临界失稳速度, 说明 旋翼的非定常气动力对倾转旋翼机的前飞稳定性造成 重要的不利影响, 如不采用非定常气动力计算, 会造成 临界失稳速度的计算值偏大,带来安全隐患。

\section{6 结论}

采用较接式旋翼、刚性短舱与弹性机翼的结构模 型和ONERA 非定常气动力模型, 并考虑翼型压缩性和 失速的影响，建立了用于分析倾转旋翼机前飞稳定性 的非定常气弹动力学模型. 采用Bell倾转旋翼机模型 的相关参数, 计算了各模态频率和阻尼比随前飞速度 变化, 并与现有的采用片条准定常气动力的倾转旋翼 机前飞动力学模型和NASA全尺寸半展风洞试验数据 进行对比. 结果表明采用片条准定常气动力模型的计 算结果与试验值相差较大，本文的计算结果接近试验 值, 说明在飞行过程中旋翼所处的非定常气动力环境 不可忽略. 同时本文所计算的倾转旋翼机前飞临界失 稳速度小于其余理论模型的计算结果，说明非定常气 动力对系统前飞稳定性有重要的不利影响，如不采用 非定常气动力计算，会造成临界失稳速度的计算值偏 大, 带来安全隐患.

\section{参考文献}

1 李治权, 夏品奇. 倾转旋翼机回转颤振及桨毂中心运动图像. 中国科学: 技术科学, 2014, 44: 286-294

2 Johnson W. Dynamics of tilting proprotor aircraft in cruise flight. NASA Technical Report, TN D-7677, 1974

3 Nixon M W. Parametric Studies for Tiltrotor Aeroelastic Stability in Highspeed Flight. J Am Helicopter Soc, 1993, 38: 71-79

4 Nixon M W. Aeroelastic response and stability of tiltrotors with elastically-coupled composite rotor blades. Dissertation for Doctoral Degree. Maryland: University of Maryland, 1994

5 岳海龙, 夏品奇. 倾转旋翼机前飞动力学稳定性分析. 航空动力学报, 2007, 22: 1863-1867

6 杨朝敏, 夏品奇. 倾转旋翼机前飞时机翼/短舱/旋翼耦合系统气弹稳定性分析. 中国科学: 技术科学, 2011, 41: 1322-1328

7 Singh R, Gandhi F, Paik J, et al. Active tiltrotor whirl-flutter stability augmentation using wing-flaperon and swash-plate actuation. J Aircraft, 2015, 44: 1439-1446

8 Zhang J, Smith E C. Influence of aeroelastically tailored wing extensions and winglets on whirl flutter stability. In: 2nd Asian Rotorcraft Forum. 
Tianjin, 2013. 188-200

9 Kim T, Shin S J, Kim T. Analysis of tiltrotor whirl flutter in time and frequency domain. J Mech Sci Technol, 2009, 23: 3281-3291

10 Bertin J, Smith M L. Incompressible Flow About Wings of Finite Span, Aerodynamics for Engineers. 3rd ed. Upper Saddle River: Prentice-Hall, 1998. 261-336

11 Saffman P G. Vortex Force and Bound Vorticity, Vortex Dynamics. Cambridge: Cambridge University Press, 1992. 46-48

12 Johnson W. Helicopter Theory. New York: Dover Publication, 1980. 196-198

13 Peters D A, Kim B S, Chen H S. Calculation of trim settings for a helicopter rotor by an optimized automatic controller. J Guidance Control Dyn, 1984, 7: 85-91

\title{
An improved unsteady aeroelastic model for tiltrotor aircraft in forward flight
}

\author{
LI ZhiQuan \& XIA PinQi \\ College of Aerospace Engineering, Nanjing University of Aeronautics and Astronautics, Nanjing 210016, China
}

When the tiltrotor aircraft is in forward flight, the rotor is in complex and unsteady aerodynamic environment. However, the existing aeroelastic stability models for tiltrotor aircraft mainly adopt quasi steady strip theory to calculate the aerodynamic airloads of rotor, which leads a significant error between the calculated and experimental data of aeroelastic stability. In this paper, the unsteady aeroelastic model for tiltrotor aircraft in forward flight has been presented by using the ONERA unsteady aerodynamic model and considering the influence of compressibility and stall effects of airfoil. According to the system parameters of NASA experimental data, the variations of modal frequencies and damping ratios with flight speed were calculated. The calculated results are closer to the experimental data compared with the existing theoretical model, indicating that the unsteady aeroelastic model established in this paper can more accurately describe the aeroelastic stability for tiltrotor aircraft in forward flight than the existing models.

tiltrotor aircraft, forward flight, aeroelastic stability, unsteady aerodynamic forces

doi: $10.1360 /$ N092017-00314 